\title{
Elevated levels of plasma von Willebrand factor and increased urinary albumin excretion are possible markers for endothelial dysfunction in type 2 diabetes mellitus patients
}

\author{
Umadevi B ${ }^{1}$, Suresh Nayak $B^{2, *}$ \\ ${ }^{1}$ Assistant Professor, Dept. of Physiology, Sapthagiri Institute of Medical Sciences \& Research Centre, Bengaluru, Karnataka, \\ ${ }^{2}$ Associate Professor, Dept. of Physiology, Dr. BR Ambedkar Medical College, Bengaluru, Karnataka, India
}

"Corresponding Author: Suresh Nayak B

Email: drbsnayak9106@gmail.com

Received: $4^{\text {th }}$ July, 2018

Accepted: $11^{\text {th }}$ August, 2018

\begin{abstract}
Introduction: Globally, Type 2 Diabetes Mellitus (T2DM) prevalence is increasing. Endothelial dysfunction is known to be associated with T2DM. The aim of the present study is to assess endothelial dysfunction by measuring plasma levels of von Willebrand factor and urine albumin levels in patients with type 2 diabetes mellitus and in healthy subjects.

Materials and Methods: This is a prospective, case-control study conducted in the department of physiology in association with department of Endocrinology, MS Ramaiah Memorial and Teaching Hospital, Bangalore. A total of 50 subjected were recruited into the study. Among them 25 were T2DM patients as cases and 25 age and sex matched healthy controls. Under aseptic conditions, After 12 hours of fasting $5 \mathrm{ml}$ venous blood sample is collected and plasma is separated. The separated plasma sample was used for the estimation FBS, PPBS, von Willebrand factor (vWF). Urine sample is used for the estimation of urine albumin. Anthropometric parameters like blood pressure, height, weight and BMI was measured.

Results: In the present study, systolic blood pressure, FBS, PPBS, von Willebrand factor, urine albumin levels were significantly increased in T2DM cases compared with healthy controls. Von Willebrand factor and urinary albumin levels showed a weak correlation in type $2 \mathrm{DM}$ patients $(\mathrm{r}=-0.245, \mathrm{p}=0.237)$ but such correlation was not observed in healthy subjects ( $\mathrm{r}=0.212$, $\mathrm{p}=0.310$ ).

Conclusion: In this study, von Willebrand factor(vWF) levels in plasma and urine albumin levels were increased significantly in T2DM patients compared to normals, which suggests that there is significant endothelial injury and vascular damage in T2DMPatients.
\end{abstract}

Keywords: Cardiovascular diseases, Endothelial dysfunction, Type 2 diabetes mellitus, Von Willebrand factor.

\section{Introduction}

Globally, Type 2 Diabetes Mellitus (T2DM) prevalence is increasing rapidly; it has become a major public health concern in the $21^{\text {st }}$ century. ${ }^{1}$ In 2015 , the prevalence of T2DM is 415 million adults. ${ }^{2}$ This has been projected to increase 642 million by $2040 .{ }^{3,4}$ Lowto middle income countries account for nearly $80 \%$ of total adult diabetic patients. ${ }^{5}$ According to International Diabetes Federation (IDF), an estimated of 1 in 11 adults aged 20-79 years had diabetes mellitus globally in 2015. The main reasons for the increasing epidemic of diabetes mellitus are many, including population ageing, urbanization, economic status, physical inactivity. Prevalence of diabetes mellitus is increasing rapidly, among $90 \%$ of the them affect by type 2 diabetes mellitus. ${ }^{6,7}$

Diabetes mellitus is main cause for premature illness and death worldwide. In India, type 2 diabetes mellitus accounts for 69 millions. Almost every tenth adult $(9.3 \%)$ in India is estimated to be affected by diabetes. In India, according to WHO reports, an estimated every 26 per 100,000 persons die due to diabetes. ${ }^{8,9}$ After China, India is having highest number of diabetic patients. ${ }^{1}$ By 2045 it was expected that India will have 134.3 million diabetes patients. Approximately 4.0 million people are estimated to die from diabetes in 2017 which is equivalent to one death every eight seconds. ${ }^{10}$ The risk factors for diabetes mellitus includes age, obesity, hypertension, lack of exercise, dyslipidemia and family history of diabetes mellitus. ${ }^{1,11}$

Endothelium serves as a barrier between the lumen and the vessel wall. It also provides a smooth nonthrombogenic surface. Vascular endothelium is not just a cell lining, but plays an active role via various mediators in the equilibrium of hemostasis and fibrinolysis, regulation of vessel tone and permeability, as well as synthesis of growth factors. ${ }^{12}$ Endothelial dysfunction is known to be associated with the pathophysiology of type 2 diabetes mellitus (DM). For endothelial dysfunction, multiple mechanisms are likely involved, mainly components of the insulin resistance syndrome may be involved in the development of diabetic dysfunctional endothelium. ${ }^{13,14}$ Dysfunctional endothelium plays a critical role in the development of atherosclerosis, a process that occurs prematurely and at an accelerated rate in diabetic patients. ${ }^{15}$

Several substances are produced from the vascular endothelial cells, which plays an important role in the regulation of haemostasis, some of these have been proposed as endothelial dysfunction markers. von Willebrand factor (vWF) is a glycoprotein, plays an 
significant role in homeostasis as a cofactor in platelet adhesion to the vascular subendothelium and as the carrier of blood coagulation factor VIII in plasma. ${ }^{16,17}$ The plasma levels of von Willebrand factor has been used as a marker for endothelial dysfunction and the levels were elevated significantly in type 2 diabetes mellitus patients concomitant with the development of microalbuminuria.

The major predictor of micro- and macro vascular complications of diabetes mellitus is microalbuminuria. In both types of diabetes mellitus, Microalbuminuria clusters with hypertension, retinopathy and atherosclerotic cardiovascular diseases. Studies have shown that microalbuminuria is associated with endothelial dysfunction. ${ }^{18}$ In contrast, vWF levels did not rise in those individuals with type 2 diabetes who remained normoalbuminuric throughout the period of follow-up. ${ }^{19}$ These results suggest that endothelial dysfunction occurs in parallel with the development of microalbuminuria. On the other hand, in type 1 diabetes mellitus, the increased levels of vWF preceded the Microalbuminuria development. ${ }^{20}$

There are different approaches (morphological, functional and biologic) may be used to assess endothelial function. Vascular endothelium damage is characterized by an increase of endothelium- derived regulatory proteins. Plasma markers of endothelial dysfunction such as von Willebrand factor (vWF), endothelin 1(ET 1), plasminogen 1 activator inhibitor type1 (PAI-1) may be useful to study early endothelium involvement. An increased levels of von Willebrand factor (vWF) reflects activation of or damage to endothelial cells and has also been described in association with atherosclerosis and diabetes mellitus. Plasma levels of von Willebrand factor (vWF) is thought to be more specific marker for endothelial dysfunction. ${ }^{19}$

Elevated levels of von Willebrand factor (vWF) have been detected in certain inflammatory or atherosclerotic vascular diseases and indicates a deterioration of endothelial cells. In patients with T2DM, microalbuminuria is associated with cardiovascular mortality. Microalbuminuria signals the presence of an atherogenic milieu because of its association with several risk factors for atherosclerosis. Microalbuminuria also represents early vascular (endothelial) damage. ${ }^{21}$ Von Willebrand factor (vWF) appears to be a predictive marker of diabetic nephropathy, which suggests that endothelial dysfunction precedes the onset of diabetic microangiopathy. Von Willebrand factor (vWF) expression as well as their possible association with different metabolic abnormalities present in diabetes remains poorly explained.

The aim of the present study is to assess endothelial dysfunction by measuring plasma levels of von Willebrand factor and urine albumin levels in type 2 diabetes mellitus patients and in healthy subjects.

\section{Materials and Methods}

This prospective, case-control study, done in Physiology Department in collaboration with Department of Endocrinology, MS Ramaiah Memorial and Teaching Hospital, Bengaluru. After getting permission from Institutional Ethical committee and written informed consent from study subjects, a total of 50 subjected were enrolled into the study. They were categorized as cases and controls. Among them, 25 type 2 diabetes mellitus patients were taken as cases and 25 age and sex matched healthy subjects were taken as controls. Among the 25 cases 12 (48\%) were males and $13(52 \%)$ were females. In controls, $13(52 \%)$ were males and $12(48 \%)$ were females. Age of the study subjects was 45 to 60 years and T2DM duration was 510 years were included in the study. The diagnosis of type 2 diabetes mellitus is made as per WHO criteria. Patients with a history of hypertension, renal impairment, autoimmune disorders, carebrovascular diseases, acute respiratory failure, previous vascular events (angina, myocardial infarction, acute arterial occlusion) BMI $>30$ were excluded from the study. A detailed clinical and physical examination was done for all study participants. Anthropometric parameters like blood pressure, height and weight was calculated. Under aseptic conditions, After 12 hours of fasting $5 \mathrm{ml}$ venous blood sample is collected from all subjects in vacutainers, containing $0.11 \mathrm{~mol} / \mathrm{ml}$ of trisodium citrate, anticoagulant. The blood samples were centrifuged at $2500 \mathrm{rpm}$ for 10 minutes to obtain plasma. The plasma was stored at $-20 \mathrm{C}$ for further analysis. The separated plasma sample was used for the estimation fasting blood sugar (FBS), PPBS, von Willebrand factor (vWF). Urine sample is used for the estimation of urine albumin. Blood sugar was estimated by GOD-POD method by using ERBA chemistry analyzer and von Willebrand factor by ELISA method by using mini VIDAS instrument. Urine albumin is measured by turbidometric method by using ERBA MANNHEIM.

\section{Statistical Analysis}

Data were expressed as mean \pm SD. P value $<0.05$ is considered as statistically significant. Statistical analysis was done by using SPSS 20.0, Stata 8.0, Microsoft word and Excel for graphs.

\section{Results}

In the present study, systolic blood pressure, FBS, PPBS, von Willebrand factor, urine albumin levels were significantly elevated in T2DM patients compared with controls. The basic characteristics between cases and controls was done by two tailed independent $t$ test. There is no significant difference between cases and controls in respect to basic characteristics like age, gender, height, weight, and BMI ( $p$ > 0.05) (Tables 1). Comparison of study parameters between cases and controls and correlation with von Willebrand factor is shown in table 2, 3 and Fig. 1. 
von Willebrand factor and urinary albumin levels showed a weak correlation in T2DM patients $(r=-$
$0.245, \mathrm{p}=0.237)$ but such correlation was not found in controls ( $\mathrm{r}=0.212$, $\mathrm{p}=0.310$ ) (Fig. 2)

Table 1: Comparison of anthropometric parameters between T2DM cases and controls

\begin{tabular}{|l|c|c|c|}
\hline \multicolumn{1}{|c|}{ Parameters } & $\begin{array}{c}\text { Cases }(\mathbf{n = 2 5}) \\
\text { Mean } \pm \text { SD }\end{array}$ & $\begin{array}{c}\text { Controls }(\mathbf{n = 2 5}) \\
\text { Mean } \pm \text { SD }\end{array}$ & p-Value \\
\hline Age & $57.16 \pm 2.61$ & $56.68 \pm 2.08$ & 0.475 \\
\hline Systolic BP $(\mathrm{mmHg})$ & $131.52 \pm 8.55$ & $124.00 \pm 7.66$ & $0.002 * *$ \\
\hline Diastolic BP $(\mathrm{mmHg})$ & $76.56 \pm 6.36$ & $76.40 \pm 7.81$ & 0.937 \\
\hline BMI $\left(\mathrm{kg} / \mathrm{m}^{2}\right)$ & $25.92 \pm 3.35$ & $25.41 \pm 2.69$ & 0.558 \\
\hline
\end{tabular}

** Highly significant $\mathrm{P}<0.01$

Table 2: Comparison of study parameters between T2DM cases and controls

\begin{tabular}{|l|c|c|c|}
\hline \multicolumn{1}{|c|}{ Parameters } & $\begin{array}{c}\text { Cases }(\mathbf{n}=\mathbf{2 5}) \\
\text { Mean } \pm \text { SD }\end{array}$ & $\begin{array}{c}\text { Controls }(\mathbf{n}=\mathbf{2 5}) \\
\text { Mean } \pm \text { SD }\end{array}$ & p-Value \\
\hline FBS (mg/dL) & $165.33 \pm 46.38$ & $74.00 \pm 7.21$ & $<0.001^{* *}$ \\
\hline PPBS $(\mathrm{mg} / \mathrm{dL})$ & $247.11 \pm 90.09$ & $98.56 \pm 4.39$ & $<0.001^{* *}$ \\
\hline Von Willebrand factor $(\mathrm{IU} / \mathrm{ml})$ & $0.99 \pm 0.18$ & $0.57 \pm 0.25$ & $\mathrm{P}<0.001^{* *}$ \\
\hline Urine Albumin $(\mathrm{mg} / \mathrm{L})$ & $73.20 \pm 20.03$ & $21.28 \pm 5.55$ & $\mathrm{P}<0.001^{* *}$ \\
\hline
\end{tabular}

** Highly significant $\mathrm{P}<0.01$

Table 3: Correlation of von Willebrand factor with Age, BMI, duration, urine albumin in T2DM cases and controls

\begin{tabular}{|l|c|c|c|c|}
\hline \multirow{2}{*}{ Parameters } & \multicolumn{2}{c|}{ Cases } & \multicolumn{2}{c|}{ Controls } \\
\cline { 2 - 5 } & r value & p value & r value & p value \\
\hline Age & 0.114 & 0.556 & -0.161 & 0.441 \\
\hline BMI & 0.231 & 0.266 & 0.023 & 0.921 \\
\hline Duration of T2DM & 0.104 & 0.620 & - & - \\
\hline Urine albumin & -0.245 & 0.237 & 0.212 & 0.310 \\
\hline
\end{tabular}

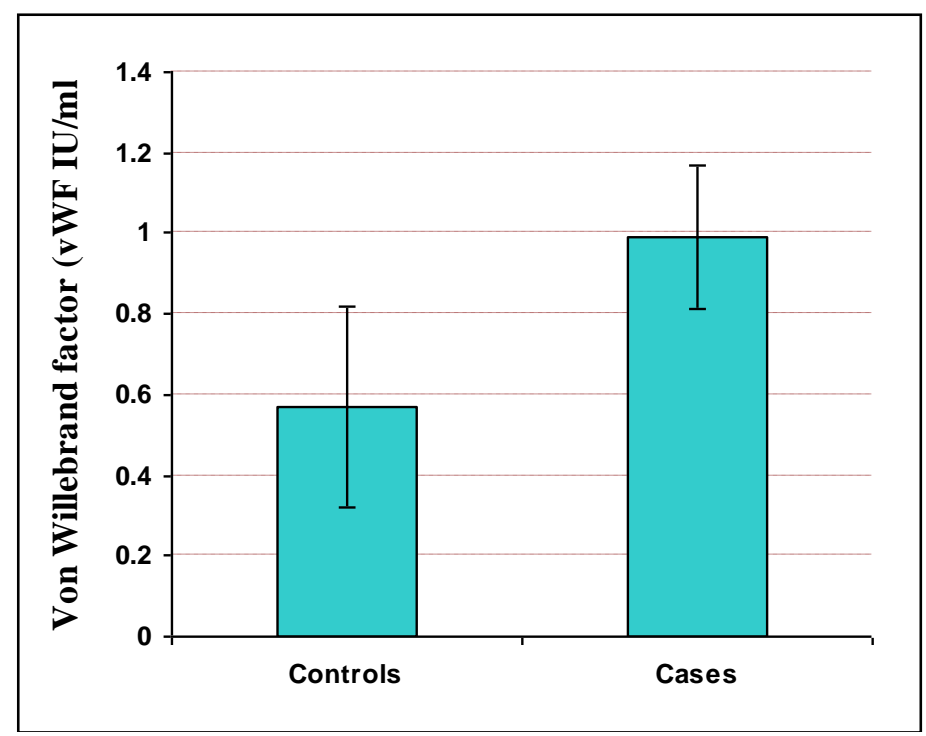

Fig. 1: Comparison of von Willebrand factor (IU/mL) between cases and controls 


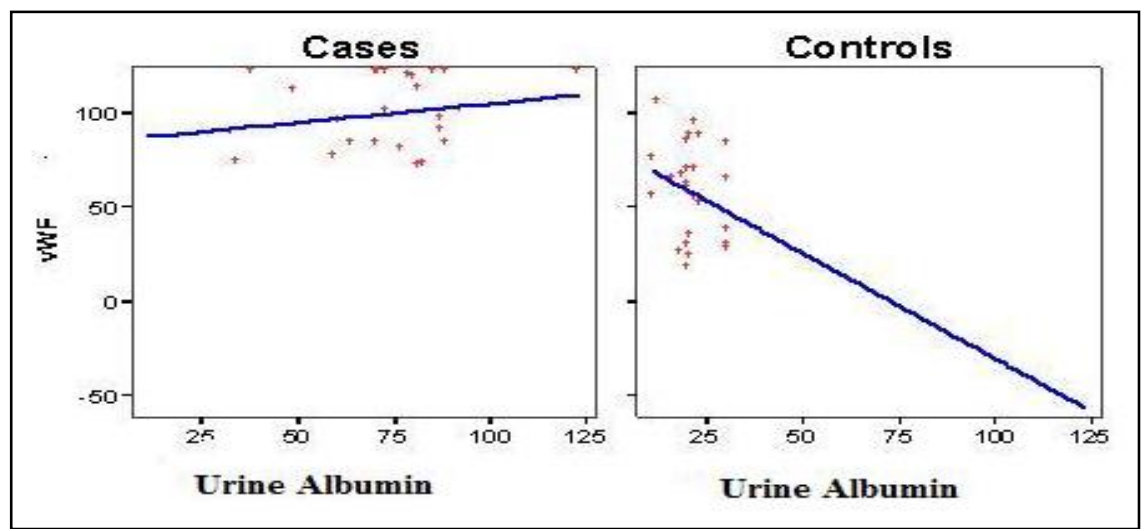

Fig. 2: Correlation between von Willebrand factor and urine albumin

\section{Discussion}

In patients with diabetes mellitus, the endothelial dysfunction causes damage to the arterial wall, atheroma, and formation of platelet microaggregates. Dyslipidemia, insulin resistance, and increased oxidative stress are major factors underlying the endothelial damage in diabetes mellitus. ${ }^{22,23}$ Dysfunctional endothelial may precede the development of atherosclerosis in the presence of different risk factors. Vascular endothelial damage is characterized by an increase of endothelium-derived regulatory proteins. The Endothelial dysfunction markers such as plasma von Willebrand factor (vWF), cellular adhesion molecules, endothelin 1(ET 1) may be useful to investigate early endothelium involvement. von Willebrand factor is thought to be more specific marker for endothelial dysfunction.

In this study, systolic blood pressure, fasting blood sugar, post-prandial blood sugar, von Willebrand factor (vWF) and urine albumin levels were significantly elevated in T2DM cases when compared to controls $(\mathrm{P}<0.001)$. This study results supports, research hypothesis that increased levels of plasma vWF and increased levels of urine albumin excretion, and their weak correlation in type 2 diabetes mellitus patients when compared to controls. Elevated levels of plasma von Willebrand (vWF), a marker of atherosclerotic and atherothrombotic burden in type 2 diabetic patients, proves that there is an endothelial dysfunction. These findings are supported by earlier studies. ${ }^{24-26}$ Thus, the elevated levels of von Willebrand factor (vWF) suggests endothelial damage, furthermore elevated levels of vWF predicts cardiovascular morbidity and mortality. ${ }^{27,28}$

von Willebrand factor (vWF) is a glycoprotein produced by vascular endothelial cells, is present in plasma, platelets, endothelial cells and also in the subendothelial matrix of the vessel wall. von Willebrand factor mediates platelet adhesion to injured endothelium, the first step in thrombus formation and it also serves as the carrier protein for coagulation factor VIII. Plays an important role in thrombosis, as well as the possibility that $\mathrm{vWF}$ could serve as a biomarker of endothelial damage. ${ }^{29}$ The main physiological function of $\mathrm{vWF}$ is to maintain hemostatic balance in the vasculature, but because the endothelium is a main source of vWF, increased levels reflect stimulation or injury to endothelial cells. It plays a significant role in adhesion of platelets to the sub-endothelium and platelet aggregation, which are the initial stages in thrombus formation.

Hyperglycemia may lead to intracellular changes in the redox state resulting in depletion of the cellular NADPH pool. Increased expression of growth factors has also been implicated in diabetes mellitus with proliferation of both endothelial cells and vascular smooth muscle, possibly promoting neovascularization. This can also lead to increased blood levels of von Willebrand factor (vWF), PAI-1, type IV collagen. In diabetes mellitus, there is an increased oxidative stress and high levels of oxidized lipoproteins, mainly small, dense low-density lipoprotein (LDL).$^{30,31}$

Patient included in this study did not have diabetic complications or any other apparent risk factors like hypertension or smoking for endothelial damage apart from diabetes. Even though the selection process intended to include only patients with these characteristics, higher levels of systolic BP, although within normal range were observed in patients with diabetes, which could reflect the phenotypic characteristics of diabetes mellitus, probably because of the insulin resistance involved in diabetes. ${ }^{27}$

Microalbuminuria, regarded as a risk indicator rather than a risk factor and is a marker of widespread vascular damage. It is a predictor of progression of diabetic nephropathy and also powerful independent risk factor for CAD. Urine albumin levels, a strong predictor for endothelial dysfunction were assessed and their correlation with von Willebrand factor (vWF) were studied in patients with T2DM and normal subjects. Urine albumin levels were significantly increased $(\mathrm{P}<0.001 * *)$ in $\mathrm{T} 2 \mathrm{DM}$ cases compared to controls. Elevated levels of plasma vWF and urine albumin reflect underlying endothelial dysfunction and weak correlation that we observed between the levels of these markers support the view that they are under 
substantially different regulatory control. The present study shows that T2DM patients have significant endothelial injury as assessed by significantly increased levels of plasma von Willebrand factor (vWF) and urine albumin excretion. These patients are probably at risk of developing cardiovascular complications in the future. Therefore early intervention is required to help these patients to prevent future cardiovascular and renal complications.

\section{Conclusion}

Globally, Type 2 Diabetes Mellitus (T2DM) prevalence is increasing rapidly; it has become a major health concern in 21 st century. In this study, plasma von Willebrand factor (vWF) and urine albumin levels were significantly elevated in T2DM patients compared to normals, which suggests that there is significant endothelial injury and vascular damage in T2DM Patients. Further large scale studies are required to understand the exact mechanism of endothelial injury. The main limitations of the study are less sample size.

\section{Conflict of Interest: Nil}

\section{Acknowledgements: Nil}

\section{References}

1. Cheng Hu and Weiping Jia. Diabetes in China: Epidemiology and Genetic Risk Factors and Their Clinical Utility in Personalized Medication. Diabetes. 2018;67:3-11.

2. Global reports on diabetes- World Health Organization. 2016:1-88.

3. Yan Zheng, Sylvia H. Ley and Frank B. Hu. Global aetiology and epidemiology of type 2 diabetes mellitus and its complications. 2018;14:88-98.

4. International Diabetes Federation. IDF Diabetes Atlas 7th Edition. Diabetes Atlas http://www.diabetesatlas.org/ (2015).

5. International Diabetes Federation (IDF. IDF Diabetes Atlas. 6th ed. 2013. Available from:

http://www.diabetesatlas.org/. [Last accessed on 2016 May 11].

6. Holman, N., Young, B. \&Gadsby, R. Current prevalence of type 1 and type 2 diabetes in adults and children in the UK. Diabetic Medicine. 2015;32:1119-1120.

7. Bruno, G. Incidence of type 1 and type 2 diabetes in adults aged 30-49 years: the population based registry in the province of Turin, Italy. Diabetes Care. 2005;28:2613-2619.

8. Saddaf Naaz Akhtar, Preeti Dhillon. Prevalence of diagnosed diabetes and associated risk factors: Evidence from the large-scale surveys in India. Journal of Social Health and Diabetes. 2017;5:28-36.

9. V. Mohan, S. Sandeep, R. Deepa, B. Shah \& C. Varghese. Epidemiology of type 2 diabetes: Indian scenario. Indian Journal Medical Research. 2007;125:217-230.

10. Medha Mathur, Navgeet Mathur, Omveer Singh, Jitendra Solanki, Pradeep Soni, Ashutosh Sarwa, Sawai Khatri et al. Demographic characters and factors favouring emergence of diabetes mellitus type two. International
Journal of Research in Medical Sciences. 2018;6(3):950954.

11. Rawat N, Mathur N, Harikrishnan R, Choudhary J, Rawat K, Mathur M. A study of correlation of serum ferritin with glycatedhaemoglobin in diabetes mellitus type 2 patients: a case control study. Asian Pacific Journal of Health Science. 2016;3(4):83-88.

12. Chong A.Y, Blann A.D, Lip G.Y.H. Assessment of endothelial damage and dysfunction: observations in relation to heart failure. QJM: An International Journal of Medicine. 2003;96:253-267.

13. Beatriz G.S. Seligman, Andreia Biolo, Carisi A. Polanczyk, Jorge L. Gross, Nadine Clausell. Increased Plasma Levels of Endothelin 1 and von Willebrand Factor in Patients with Type 2 Diabetes and Dyslipidemia. Diabetes Care. 2000;23:1395-1400.

14. Fagan TC, Deedwania PC: The cardiovascular dysmetabolic syndrome. American Journal of Medicine. 1998;105(Suppl.1A):77S-82S.

15. Ross R. The pathogenesis of atherosclerosis: a perspective for the 1990s. Nature. 1993;362:801-809.

16. Nervana Samy, Mie Afify, Nabila Abd E Maksouda, Magda Sayeda, Azza Imam. Circulating markers of endothelial dysfunction in type 2 diabetic patients with Microalbuminuria. Asian Biomedicine. 2012;6(2):175183.

17. Ruggeri ZM, Ware J. Von Willebrand factor. Faseb Journal. 1993;7:308-316.

18. Peter Gaede, pernille Vedel, Hans-Henrik Parving and Oluf Pedersen. Elevated levels of plasma Von Willebrand factor and the risk of macro and microvascular disease in type 2 diabetic patients with Microalbuminuria. Nephrology Dialysis Transplantation. 2001;16:20282033.

19. Pier Mannuccio Mannucci. von Willebrand Factor, A Marker of Endothelial Damage? Arteriosclerosis, Thrombosis \& Vascular Biology. 1998;18:1359-1362.

20. Meyer D, Girma J P. Von Willebrand factor: structure and function. Thrombosis \& Haemostasis. 1993;70:99104.

21. Piero O. Bonetti, Lilach O. Lerman, Amir Lerman. Endothelial Dysfunction. A Marker of Atherosclerotic Risk. Arteriosclerosis, Thrombosis \& Vascular Biology. 2003;23:168.

22. Tamer H. El-Sersy. Evaluation of Von Willebrand factor as an early detector of diabetic retinopathy. Journal of Egyptian Ophthalmological Society. 2014;107:209-213.

23. Hink U, Li H, Mollnau H. Mechanisms underlying endothelial dysfunction in diabetes to mellitus. Circulation Research. 2001;88:14-22.

24. Meigs JB, O'donnell CJ, Tofler GH, Benjamin EJ, Fox CS, Lipinska I, Nathan DM, Sullivan LM, D'Agostino RB, Wilson PW. Hemostatic markers of endothelial dysfunction and risk of incident type 2 diabetes: the Framingham Offspring Study. Diabetes. 2006;55:530537.

25. Jensen T. Increased plasma level of von Willebrand factor in Type1 (insulin-dependent) diabetic patients with incipient nephropathy. British Medical Journal. 1989;298:27-28.

26. Shimano H, Takahashi K, Kawakami M. Elevated serum and urinary thrombomodulin levels in patients with noninsulin-dependent diabetes mellitus. Clinica Chimica Acta. 1994;25:89-96.

27. Seligman BG, Biolo A, Polanczyk CA, Gross JL, Clausell N. Increased plasma levels of endothelin 1 and von Willebrand factor in patients with type 2 diabetes and dyslipidemia. Diabetes Care. 2000;(9):1395-400. 
28. Cesari M, Penninx BW, Newman AB, Kritchevsky SB, Nicklas BJ, Sutton-Tyrrell K, Rubin SM, Ding J, Simonsick EM, Harris TB, Pahor M. Inflammatory markers and onset of cardiovascular events: results from the Health ABC study. Circulation. 2003;108(19):23172322.

29. David S. Frankel, James B. Meigs, Joseph M. Massaro, Peter W.F. Wilson, Christopher J. O’Donnell, Ralph B. D' Agostino, Geoffrey H. Tofler. Von Willebrand Factor, Type 2 Diabetes Mellitus, and Risk of Cardiovascular Disease. The Framingham Offspring Study. Circulation. 2008; 118:2533-2539.

30. Ibrahim HA, Melig AA. El-I Abdel-Hamid, Elhendy MA. Relations between von Willebrand factor, markers of oxidative stress and microalbuminuria in patients with type 2 diabetes mellitus, Medical Science Monitor. 2004;10:85-89.
31. Giancarlo DM, Maria CB, Oriana L, Arianna M, Rosalba C, Alessandra G, et al. Endothelial dysfunction and oxidative stress in type 1 and type 2 diabetic patients without clinical macrovascular complications. Diabetes Research and Clinical Practice. 2008;79:337-342.

How to cite this article: Umadevi B, Nayak S.B. Elevated levels of plasma von Willebrand factor and increased urinary albumin excretion are possible markers for endothelial dysfunction in type 2 diabetes mellitus patients. Indian $\mathrm{J}$ Clin Anat Physiol. 2018;5(4):468-473. 